\title{
Canal nutrício mimetizando reabsorção radicular externa
}

Carolina Cintra Gomes ${ }^{1}$, Diogo Rodrigues Cruvinel2 ${ }^{2}$ Brunno Santos de Freitas Silva ${ }^{3}$, Rogério Ribeiro de Paiva ${ }^{4}$, Mayara Barbosa Viandelli Mundim-Picoli ${ }^{5}$, Helder Fernandes de Oliveira ${ }^{6}$, Giulliano Caixeta Serpa ${ }^{7}$

${ }^{1}$ Doutora em Radiologia Odontológica; Professora Titular da área de Diagnóstico e Cirurgia Oral do Curso de Odontologia do Centro Universitário UniEVANGÉLICA. ${ }^{2}$ Doutor em Odontologia; Professor Titular da área de Reabilitação Oral do Curso de Odontologia do Centro Universitário UniEVANGÉLICA. ${ }^{3}$ Doutor em Patologia Bucal; Professor Titular da área de Diagnóstico e Cirurgia Oral do Curso de Odontologia do Centro Universitário UniEVANGÉLICA. ${ }^{4}$ Doutor em Ciências da Saúde; Professor Titular da área de Diagnóstico e Cirurgia Oral do Curso de Odontologia do Centro Universitário UniEVANGÉLICA. ${ }^{5}$ Doutora em Odontologia; Professora Adjunto da área de Diagnóstico e Cirurgia Oral do Curso de Odontologia do Centro Universitário UniEVANGÉLICA. ${ }^{6}$ Doutor em Odontologia; Professor Titular da área de Endodontia do Curso de Odontologia do Centro Universitário UniEVANGÉLICA. ${ }^{7}$ Mestre em Odontologia; Professor Adjunto da área de Endodontia do Curso de Odontologia do Centro Universitário UniEVANGÉLICA.

\begin{abstract}
Resumo
Introdução: A reabsorção radicular externa (RRE) é um processo patológico decorrente do dano ou da remoção da camada cementoblástica superficial, conjuntamente, ou não, a perda substancial da porção dentinária radicular. Acredita-se que a RRE em dentes permanentes resulta de fatores mecânicos ou inflamatórios, forças ortodônticas, traumatismo dentário, cistos ou tumores, periodontite crônica periapical e deficiência na regeneração do periodonto no reimplante ou transplante dentário. Objetivo: O objetivo deste relato de caso é apresentar para o cirurgião-dentista a importância da tomografia computadorizada de feixe cônico (TCFC) na investigação de imagem suspeita de RRE. Relato do caso: Paciente B.A.F., 72 anos, gênero feminino, compareceu à Clínica Odontológica de Ensino da UniEVANGÉLICA com a seguinte queixa principal: "Quero trocar minha prótese". Após exame clínico foram realizadas radiografias periapicais dos dentes remanescentes suspeitando-se de RRE no terço apical de dente 31. Realizou-se então teste de sensibilidade pulpar que apresentou resposta positiva. Na história odontológica pregressa a paciente negou presença de sintomatologia dolorosa, traumatismo dentário ou tratamento ortodôntico. Para esclarecer a suspeita, indicou-se a TCFC por meio da qual se descartou a hipótese de RRE. Verificou-se então a presença de um canal nutrício na região distal do dente 31, que na imagem periapical formou uma imagem radiolúcida que mimetizou a RRE. Considerações finais: Acredita-se que quando a RRE é confirmada, o cirurgião-dentista deve avaliar criteriosamente e considerar o tratamento endodôntico, restaurador, ressecção da raiz envolvida e exodontia, se necessário. Sendo assim, a TCFC foi essencial para o correto diagnóstico impedindo um procedimento invasivo desnecessário.
\end{abstract}

Palavras-chave: Reabsorção da raiz; Radiografia dentária; Tomografia computadorizada de feixe cônico.

\section{Introdução}

A reabsorção radicular externa (RRE) é um processo patológico decorrente do dano ou da remoção da camada cementoblástica superficial, conjuntamente, ou não, a perda substancial da porção dentinária radicular ${ }^{1}$. Esta lesão pode apresentar diversos fatores etiológicos, como: A pressão exercida pela presença de um cisto adjacente ao dente, granuloma periapical, neoplasia, raízes justapostas e dente impactado, dentre outros ${ }^{2}$. O tratamento endodôntico não é indicado em um dente com RRE extensa, sendo a exodontia geralmente empregada nos dentes afetados por essa condição ${ }^{3}$.

Canais nutrícios são estruturas anatômicas presentes no osso alveolar que representam a continuação do canal da mandíbula. Esses canais são derivados do ramo incisivo do nervo alveolar inferior, sendo atribuídos a eles a função de promover um aporte nutrício entre os elementos neurovasculares e os dentes, como também, as suas estruturas adjacentes. Em radiografias periapicais essas composições são observadas como imagens radiolúcidas lineares entre os incisivos inferiores, que por vezes, podem ser de difícil identificação $0^{4,5}$.

Um estudo recente demonstrou que a detecção dos canais nutrícios é extremamente favorecida pelo emprego de exames de $\mathrm{TCFC}^{5}$.

$\mathrm{Na}$ endodontia o diagnóstico, o plano de tratamento e a proservação são realizados rotineiramente com a ajuda de radiografias convencionais. A imagem radiográfica é essencialmente uma imagem bidimensional de um objeto tridimensional (3D) e sua interpretação pode ser influenciada pela sobreposição das estruturas anatômicas. Além disso, deve-se considerar que as estruturas visualizadas estão sujeitas a distorções geométricas ${ }^{6}$. Esses problemas podem ser superados pelo uso de imagens tridimensionais (3D) por meio da TCFC.

O diagnóstico de reabsorção radicular é desafiador. As radiografias muitas vezes não conseguem detectar reabsorções radiculares ao longo da face lingual dos dentes. Além disso, o efeito "Burnout", que é característico das radiografias periapicais, dificulta o diagnóstico dessa lesão do órgão dentário no terço cervical. As imagens de TCFC têm sido utilizadas no diagnóstico e acompanhamento das $R_{R E}$. A partir dessas imagens pode-se detectar a natureza e a localização do defeito proveniente da reabsorção. As estratégias de tratamento podem ser melhor planejadas com base nas imagens 3D desses defeitos ${ }^{6}$.

Portanto, este trabalho teve o objetivo de apresentar um caso clínico em que a utilização da TCFC foi determinante para a diferenciação entre um canal nutrício e uma alteração suspeita de RRE. 


\section{Relato de caso}

Uma paciente do gênero feminino, 72 anos de idade, gênero feminino, foi examinada na Clínica Odontológica de Ensino da UniEVANGÉLICA com a seguinte queixa principal: "Quero trocar minha prótese". Durante o exame clínico não foram constadas alterações sistêmicas contributórias para o caso em questão. Após este exame foram realizadas radiografias periapicais dos dentes remanescentes para a avaliação pré-protética.

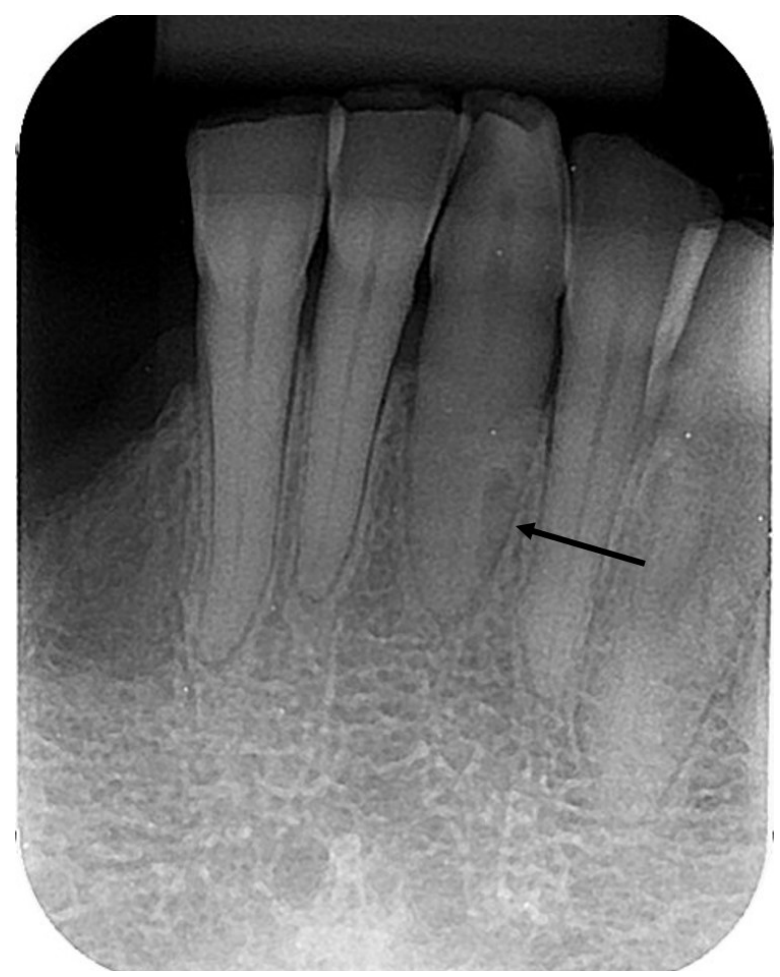

Figura 1 - Radiografia periapical onde se observa imagem radiolúcida no terço apical da raiz do dente 31 , apresentando como hipótese de diagnóstico reabsorção radicular externa.

Na radiografia periapical de incisivos inferiores foi observada imagem radiolúcida, de formato semi-circunferencial, no terço apical da raiz do dente 31, apresentando uma grande similaridade com uma RRE (Figura 1). Realizou-se então teste de vitalidade pulpar a frio no dente em questão apresentando resposta positiva. Na história odontológica pregressa a paciente negou presença de sintomatologia dolorosa, traumatismo dentário ou tratamento ortodôntico prévio, e na história médica pregressa sem alterações significativas.

Frente a ausência de informações que pudessem categoricamente indicar a presença de uma RRE de origem inflamatória, constatou-se a necessidade de realização de um exame de TCFC afim identificar a relação da lesão com as estruturas do dente 31, como também, averiguar a sua relação com as estruturas ósseas adjacentes. $\mathrm{O}$ exame tridimensional revelou a presença de um canal nutrício na região distal do dente 31, que na imagem periapical formou uma imagem radiolúcida que mimetizou a RRE (Figura 2). Logo, considerando o exame clínico e radiográfico dentro dos padrões de normalidade, a paciente foi encaminhada para a reabilitação protética.

\section{Discussão}

As informações de diagnóstico são essenciais para minimizar erros e apoiar a tomada de decisões e o planejamento apropriado. Imagens precisas permitem um melhor planejamento do tratamento e potenciali-

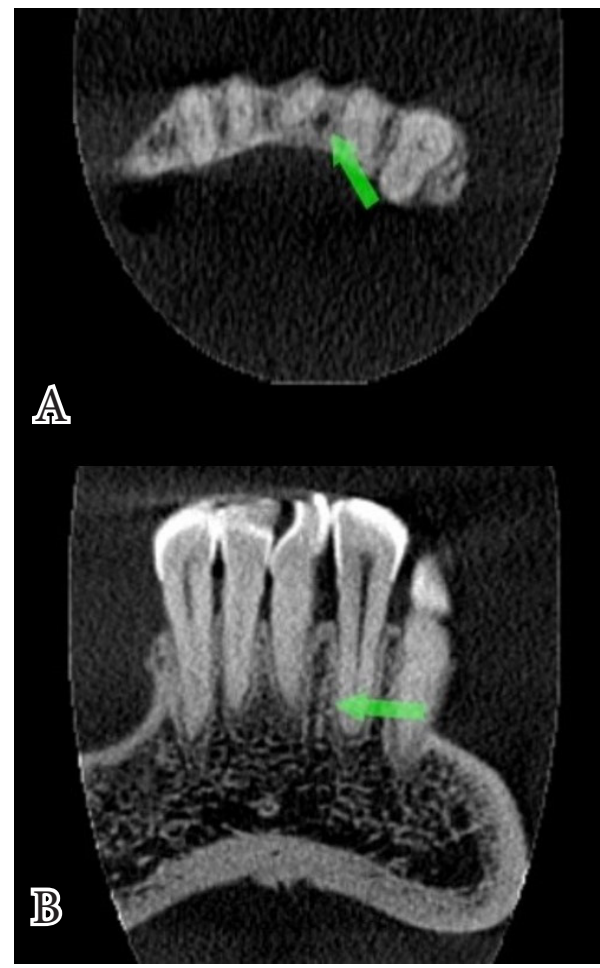

Figura 2 - Imagens por TCFC elucidando a presença de canal nutrício entre os dentes 31 e 32 . (A) Corte axial. (B) Corte coronal.

zam resultados mais previsíveis e adequados ${ }^{8-10}$. Neste estudo, o uso da TCFC foi conveniente e permitiu a visualização das estruturas adjacentes à região suspeita, elucidando a presença de um canal nutrício paralelo ao terço apical do dente em questão.

Os canais nutrícios são considerados pequenos vasos sanguíneos, também denominados ramos peridentais, provenientes da anastomose entre os ramos milo-hióideo direito e esquerdo da artéria alveolar inferior que ocorre no plano mediano na mandíbula ${ }^{11}$. Radiograficamente são vistos como linhas radiolúcidas, que correspondem aos trajetos intra-ósseos das arteríolas ou vênulas. Podem ser observados em radiografias oclusais, periapicais e panorâmicas, sendo mais prevalentes na região de seios maxilares, dentes incisivos inferiores e em áreas edêntulas com pouca espessura óssea ${ }^{12}$

No presente caso a imagem radiolúcida descrita como canal nutrício foi observada com formato semi-circunferencial, no terço apical da raiz do dente 
31, apresentando uma grande similaridade com uma RRE. As radiografias convencionais apresentam como desvantagem a sobreposição de imagens, por serem imagens bidimensionais, podendo em algumas situações, haver confusão entre estruturas anatômicas e processos patológicos. Portanto, o conhecimento da anatomia normal e dos sinais clínicos da hipótese diagnóstica em questão devem ser considerados para se indicar os exames complementares, quando necessários, e se estabelecer o diagnóstico final.

\section{Considerações finais}

Os exames por imagem são grandes aliados na definição do diagnóstico na Odontologia. A TCFC é um exame no qual a anatomia pode ser analisada detaIhadamente, e nesse caso foi esclarecedor em relação à suspeita inicial de RRE.

\section{Referências}

1. Ahangari Z, Nasser M, Mahdian M, Fedorowicz Z, Marchesan MA. Interventions for the management of external root resorption. Cochrane Database Syst Rev. 2015 Nov 24;(11):CD008003.

2. Benenati FW. Root resorption: Types and treatment. Gen Dent. 1997;45:42-5.

3. Weine FS. Endodontic Therapy. 6th ed. St Louis: Mosby; 2004. pp. 1-23. (423-51)

4. Fielding CG. Nutrient canals of the alveolar process as an anatomic feature for dental identifications. J Forensic Sci. 2002 Mar;47(2):381-3.

5. Ogawa A, Fukuta $Y$, Nakasato $H$, Nakasato S. Cone beam computed tomographic evaluation of nutrient canals and foramina in the anterior region of the mandible. Surg Radiol Anat. 2016 Nov;38(9):1029-1032.

6. Cotton TP, Geisler TM, Holden DT, Schwartz SA, Schindler WG. Endodontic applications of cone-beam volumetric tomography. J Endod 2007; 33: 1121-32.

7. Maini A, Durning P, Drage N. Resorption: within or without? The benefit of cone-beam computed tomography when diagnosing a case of an internal/external resorption defect. Br Dent Three-dimensional imaging modalities in endodontics J 2008; 204: 135-7.

8. Cohenca N, Simon JH, Mathur A, Malfaz JM. Clinical indications for digital imaging in dento-alveolar trauma. Part 2: root resorption. Dent Traumatol 2007; 23: 105-13.

9. Ballal S., Sachdeva G. S., Kandaswamy D. Endodontic management of a fused mandibular second molar and paramolar with the aid of spiral computed tomography: a case report. Journal of Endodontics. 2007;33(10):1247-1251. doi: 10.1016/j. joen.2007.06.021. [PubMed] [Cross Ref]

10. Simon J. H. S., Enciso R., Malfaz J.-M., Roges R., Bailey-Perry M., Patel A. Differential diagnosis of large periapical lesions using cone beam computed tomography measurements and biopsy. Journal of Endodontics. 2006;32(9):833-837. doi: 10.1016/j.joen.2006.03.008. [PubMed] [Cross Ref]

11. Rizzolo RJC, Madeira MC. Anatomia Facial com fundamentos de anatomia geral. $5^{\text {a }}$ ed. 2016, Sarvier.

12. Renata Vasconcelos Pavan RV, Borges PR, Rezende VM, Paiva RR. Anatomia radiográfica da região bucomaxilofacial: Uma abordagem interativa na web [Internet]. Anápolis: UniEVANGÉLICA; c2002-2017 [update 2002; cited 2017 Jul 21]. UniEVANGÉLICA Centro Universitário - Curso de Graduação em Odontologia [24p] Available from: http://www.unievangelica.edu.br/gc/graduacao/odontologia/projeto/DescricaoDasEstruturas.pdf.

Recebido em : 04/08/2017

Aprovado em: 12/09/2017

Os autores declaram que não há conflitos de interesse.

\section{Autor correspondente:}

Carolina Cintra Gomes

Av. Universitária Km 3,5, Cidade Universitária - Anápolis/ GO CEP: $75083-515$

Telefone:+55 (62) 982712244

e-mail: carolcintrag@hotmail.com 


\title{
Nutrient canal resembling an external root resorption
}

\begin{abstract}
Introduction: The external root resorption (ERR) is a pathological process due to damage or removal of the superficial cementoblastic layer, jointly or not, with the substantial loss of the radicular dentin. It is believed that ERR in permanent teeth results from mechanical or inflammatory factors, orthodontic forces, dental trauma, cysts or tumors, chronic periapical periodontitis and deficiency in periodontal regeneration in cases of dental reimplantation or dental transplantation. Objective: The purpose of this case report is to present to the dentist the importance of cone beam computed tomography (CBCT) in the investigation of a suspected ERR. Case report: Patient B.A.F., 72 years old, female, attended to the UniEVANGÉLICA Dental Clinic with the following main complaint: "I want to change my prosthesis." After clinical examination, periapical radiographs of the remaining teeth were performed, suspecting of ERR in the apical area of the tooth 31. In the previous dental history, the patient denied the presence of painful symptoms, dental trauma or orthodontic treatment. In order to clarify the suspicion, it was indicated the $\mathrm{CBCT}$, which discarted the initial hypothesis of ERR.The presence of a nutrient canal in the distal region of the tooth 31 was verified, which in the periapical image formed a radiolucent image that mimicked the ERR. Final considerations: It is believed that when the ERR is confirmed, the dentist should evaluate and consider the treatment possibilities as endodontic treatment, restorative treatment, root resection and extraction, if necessary. Thus, CBCT was essential for the correct diagnosis, preventing an unnecessary invasive procedure.
\end{abstract}

Keywords: Root Resorption; Radiography, Dental; Cone-Beam Computed Tomography. 